\title{
Role of HuR in keratinocyte migration and wound healing
}

\author{
DAVID C. BOSANQUET, LIN YE, KEITH G. HARDING and WEN G. JIANG
}

Departments of Surgery and Wound Healing, Cardiff University School of Medicine, Heath Park, Cardiff CF14 4XW, UK

Received August 24, 2011; Accepted November 3, 2011

DOI: $10.3892 / \mathrm{mmr} .2011 .675$

\begin{abstract}
Human antigen R (HuR) is a post-translational modifier of mRNAs rich in AU- and U-rich elements. These mRNAs typically code for proteins involved in cell growth and differentiation, signal transduction, transcriptional and translational control, apoptosis, nutrient transport and metabolism. Thus, HuR affects a variety of biological functions and processes. Via its effect on growth and cellular migration, HuR has been shown to enhance clinical progression of a number of cancers. Its role in wound healing remains unknown. In the present study, we evaluated HuR tissue expression in a cohort of chronic healed and non-healed leg ulcers. We also evaluated the effect of HuR knockdown on a number of cellular processes using the HaCaT human keratinocyte cell line. HuR was expressed in greater levels in the 'chronic healed' cohort of ulcers, compared to the 'chronic non-healed', although this failed to reach statistical significance $(\mathrm{p}=0.13)$. HuR knockdown resulted in greater cellular growth, faster progression through the cell cycle and reduced apoptosis. Furthermore, it reduced cellular adhesion rates without affecting migration. We, therefore, concluded that HuR promotes wound healing, primarily through its effect on cellular adhesion. It also slows cellular growth rate via its effect on both cell cycle progression and rates of apoptosis.
\end{abstract}

\section{Introduction}

Human antigen $\mathrm{R}$ (HuR) was first cloned and identified as a member of the Hu family in 1996 (1); it was localised to chromosome 19 a year later (2). The Hu family is composed of the neuronal $\mathrm{Hu}$ proteins $\mathrm{HuB}, \mathrm{HuC}$ and $\mathrm{HuD}$, as well as the ubiquitously expressed protein $\mathrm{HuR}$ (2). The three neuronal $\mathrm{Hu}$ proteins, only expressed in differentiated neurons, play important roles in neuronal plasticity and differentiation. In humans, HuR is widely expressed in all proliferating cells. The Hu protein family shares homology with the Drosophila

Correspondence to: Dr D.C. Bosanquet, Departments of Surgery and Wound Healing, Cardiff University School of Medicine, Heath Park, Cardiff CF14 4XW, UK

E-mail: davebosanquet@hotmail.com

Key words: human antigen $\mathrm{R}$, wound healing, keratinocyte migration, $\mathrm{HaCaT}$ embryonic lethal abnormal vision (ELAV) proteins, and are thus occasionally referred to as the Hu/ELAV family (3). The HuR protein contains three RNA recognition motifs (RRMs 1-3) which share a $>90 \%$ amino acid sequence identity with other Hu family members (4). RRM1 and 2 bind to the AU- and U-rich elements (also known as AREs) of mRNA, while RRM3 is thought to bind to the poly A tail and promote stability of the protein/mRNA complex (5-7). HuR also contains a variable basic hinge region between RRM2 and RRM3, which contains the HuR nucleo-cytoplasmic shuttling sequence (HNS). This sequence allows HuR to shuttle mRNA between the nucleus and cytoplasm (8). It is this ability of HuR to transport mRNAs rich in AREs, and thus alter their expression, which allows it to affect a number of biological functions. Many ARE-rich mRNAs encode functionally diverse proteins, including proteins regulating cell growth and differentiation, signal transduction, transcriptional and translational control, haematopoiesis, apoptosis, nutrient transport and metabolism (9). A number of these functions contribute to carcinogenesis, and elevated HuR levels have been associated with a poorer prognosis in colonic (10), breast $(11)$, ovarian $(12,13)$ and gastric carcinoma (14) (reviewed in ref. 15).

Wound healing is a complex co-ordinated response to injury, which is divided into four overlapping phases: haemostasis, inflammation, proliferation and maturation (16). Failure or prolongation of one or more phases results in either healing delay or non-closure of the wound. Chronic wounds present a serious problem to both the patient and the physician - they are a source of much morbidity and even mortality, they are costly to treat and can be difficult to manage (17). The dynamic cellular events during the process of wound healing requires co-ordinated gene and protein expression at a time- and location-dependent manner. Thus, regulation of mRNA, the key function of HuR, during the healing process is essential. HuR has not been previously studied in the context of wound healing. In this study, we firstly analysed HuR transcript levels in wound tissues, and, secondly, we evaluated the effects of HuR knockdown on cellular functions using the human keratinocyte 'HaCaT' cell line.

\section{Materials and methods}

Cells and tissues. The HaCaT human keratinocyte cell line was purchased from the German Cancer Institute. These cells were routinely cultured in DMEM/Ham's F12 with L-glutamine medium (PAA Laboratories, Somerset, UK), supplemented with streptomycin, penicillin and $10 \%$ foetal calf serum 
Table I. Primer sequences for conventional and quantitative PCR.

Sense primers $\left(5^{\prime}-3^{\prime}\right)$

Antisense primers (5'-3')

$\begin{array}{ll}\text { HuR (PCR) } & \text { GAGCTCAGAGGTGATCAAAG } \\ \text { HuR (Q-PCR) } & \text { AGACATGTTCTCTCGGTTTG } \\ \text { GAPDH (PCR) } & \text { GGCTGCTTTTAACTCTGGTA } \\ \text { GAPDH (Q-PCR) } & \text { CTGAGTACGTCGTGGAGTC }\end{array}$

(PAA Laboratories), and incubated at $37^{\circ} \mathrm{C}$ in $5 \% \mathrm{CO}_{2}$ and 95\% humidity. Human wound tissues were collected from patients who attended wound clinics at the University Hospital of Wales, with the approval of the South East Wales Local Research Ethics Committee. Core biopsies were taken from the wound edge, and were immediately stored at $-80^{\circ} \mathrm{C}$ and subsequently in liquid nitrogen, until used. The wound tissues were all chronic (present for a minimum of 3 months despite conservative treatment) and of venous ulcer aetiology. They were biopsied at the time of the visit to the clinic (time zero), after which patients were treated and followed up routinely. Wounds that healed within 3 months of their initial visit were classified as 'Chronic healed', whereas those which failed to heal within the time frame were termed 'Chronic non-healed'. The samples were anonymised before processing and only decoded after the final test. Our wound tissue cohort comprised 51 chronic non-healing wounds and 20 chronic healed wounds.

Total cellular RNA preparation. Total cellular RNA was isolated from the homogenized tissue samples and human cell lines using the TRI reagent (Sigma, Dorset, UK), according to the manufacturer's protocol. Following extraction, the RNA concentration was quantified through spectrophotometric measurement (WPA UV 1101; Biotech Photometer, Cambridge, UK).

Reverse transcription-polymerase chain reaction (RT-PCR). cDNA was generated from 250 ng of each RNA sample and reverse transcription was undertaken using an enhanced avian RT-PCR-100 kit with anchored oligo(dt) primers (Sigma). The quality of cDNA was verified using primers to detect the housekeeping gene GAPDH (full primer sequences are outlined in Table I). Conventional PCR was performed in a T-Cy Thermocycler (Creacon Technologies Ltd., CD Emmen, The Netherlands) using REDTaq ${ }^{\circledR}$ ReadyMix ${ }^{\mathrm{TM}}$ PCR Reaction Mix (Sigma).

PCR conditions were as follows: denaturing at $94^{\circ} \mathrm{C}$ for $30 \mathrm{sec}$, annealing at $55^{\circ} \mathrm{C}$ for $30 \mathrm{sec}$ and extension at $72^{\circ} \mathrm{C}$ for $30 \mathrm{sec}$. PCR was conducted over 38 cycles with an initial 5 -min denaturing step $\left(94^{\circ} \mathrm{C}\right)$ and a final 10 -min extension step $\left(72^{\circ} \mathrm{C}\right)$, and stored until required at $4^{\circ} \mathrm{C}$. The PCR products were then loaded into a $0.8 \%$ agarose gel, separated electrophoretically, stained with ethidium bromide and visualised under ultraviolet light.

Quantitative-polymerase chain reaction $(Q-P C R)$. The iCycler IQ system (Bio-Rad, Hertfordshire, UK) was used to detect and quantify the number of HuR transcripts in the wound specimens (18), as previously described $(19,20)$. The results are provided as copy number/ $\mu$ l based on an internal standard. The results were further normalised against the expression of GAPDH in these samples. The Q-PCR technique used the Amplifluor system (Intergen Inc., Purchase, NY, USA), Q-PCR Master Mix (ABgene, Surrey, UK) and a universal probe (Uniprimer $^{\mathrm{TM}}$; Intergen) to record the fluorescence emitted by the z-sequences. Conditions for Q-PCR were an initial period of $15 \mathrm{~min}$ at $95^{\circ} \mathrm{C}$, followed by 80 cycles of $95^{\circ} \mathrm{C}$ for $15 \mathrm{sec}$, $55^{\circ} \mathrm{C}$ for $60 \mathrm{sec}$ and $72^{\circ} \mathrm{C}$ for $20 \mathrm{sec}$. Full details of the primers used are outlined in Table I.

Generation of HuR knockdown in human keratinocyte cell lines. Anti-human HuR hammerhead ribozymes were designed based on the secondary structure of HuR mRNA, generated using the Zuker RNA mFold program (21). The ribozymes were synthesized and cloned into a pEF6/V5-His-TOPO plasmid vector (Invitrogen, Paisley, UK). An appropriate ribozyme transgene and empty pEF6 control plasmid were transfected into $\mathrm{HaCaT}$ cells, as previously reported $(22,23)$. Following transfection and blasticidin selection, cells were subsequently cultured in maintenance medium and routinely tested to confirm knockdown of HuR expression. HaCaT cells containing the ribozyme transgenes and the control PEF plasmids were designated as $\mathrm{HaCaT} \mathrm{HuR}$ knockdown and $\mathrm{HaCaT}$ PEF, respectively.

SDS-PAGE and Western blotting. Following lysis of cells, equal amounts of protein from each sample were separated on a 10\% acrylamide gel. Proteins were probed using the respective primary antibodies at a concentration of 1:300 (HuR) and 1:500 (GAPDH) and specific peroxidase-conjugated secondary antibodies at a concentration of 1:1,000. Protein bands were documented using a gel documentation system (UVITech, Cambridge, UK).

In vitro growth assay. Cells $(3,000)$ from the HuR knockdown and PEF control cell line were seeded into a 96-well plate in triplicate. Cells were then incubated for 1, 3 and 5 days before being fixed in $4 \%$ formaldehyde $(\mathrm{v} / \mathrm{v})$ and stained with $0.5 \%$ (w/v) crystal violet. The crystal violet stain was then extracted using $10 \%$ acetic acid (v/v) and cell density was determined by measuring the absorbance of this solution at a wavelength of $540 \mathrm{~nm}$ using an EL x800 spectrophotometer (Bio-Tek Instruments Inc., Winooski, VT, USA). Data was normalised using day 1 absorbance to account for any seeding bias.

Analysis of cell cycle. Cells $(500,000)$ were seeded into a large flask $\left(75 \mathrm{~cm}^{2}\right)$ in normal medium on day 0 . On day 1 , the normal medium was replaced with serum-free medium to 


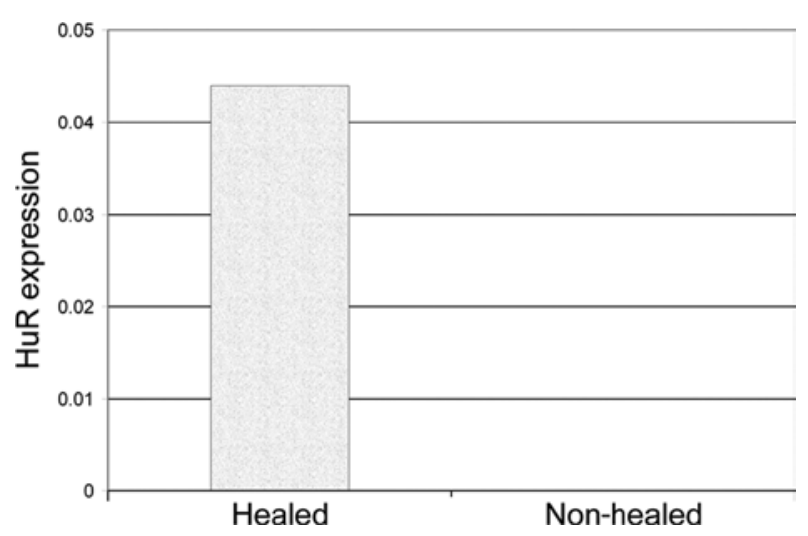

Figure 1. Levels of HuR mRNA transcript in chronic healed $(n=20)$, and chronic non-healed $(n=51)$ ulcers, normalised to GAPDH (values shown are medians). HuR expression was non-significantly higher in the healed chronic wounds [median (IQR): $0.044(0-0.16)]$, compared to the non-healed wounds [median (IQR): 0 (0-1.0), P=0.13].

arrest cellular proliferation in the G0 phase. On the evening of day 3 , the medium was changed back to normal to restart cellular proliferation. Sixteen hours later, the cells were collected, fixed in $70 \%$ ethanol at $4^{\circ} \mathrm{C}$ for $2 \mathrm{~h}$, resuspended in $1 \mathrm{ml}$ of PI staining solution, and incubated for $15 \mathrm{~min}$ at $37^{\circ} \mathrm{C}$. The samples were then analysed using the Partec CyFlow ${ }^{\circledR}$ SL flow cytometer and the accompanied FloMax software package (Partec GmbH, Munich, Germany). The cell cycle was determined using DNA content to label cells in either the $\mathrm{G} 0 / 1, \mathrm{~S}$ or $\mathrm{G} 2 / \mathrm{M}$ phase of replication.

Analysis of apoptosis. Cells (150,000 or 300,000) were seeded into $25-\mathrm{cm}^{2}$ flasks labelled normal or serum-free medium, respectively, and initially filled with normal medium. On day 1 the medium was changed in all flasks to either normal or serum-free, as appropriate. On day 4, all cells were harvested, including the cells floating in the culture medium, and resuspended in $1 \mathrm{X}$ Annexin-binding buffer at a concentration of $\sim 1 \times 10^{6}$ cells $/ \mathrm{ml}$. FITC annexin $\mathrm{V}(5 \mu \mathrm{l})$ and $1 \mu \mathrm{l}$ of the PI working solution $\left(100 \mu \mathrm{g} / \mathrm{ml}\right.$, supplied by Vybrant $\left.{ }^{\circledR}\right)$ was added to each $100 \mu \mathrm{l}$ of cell suspension, and the cells were incubated at $37^{\circ} \mathrm{C}$ for $15 \mathrm{~min}$. Annexin binding buffer $1 \mathrm{X}(400 \mu \mathrm{l})$ was then added prior to processing, which was performed using the Partec CyFlow ${ }^{\circledR}$ SL flow cytometer and the accompanied FloMax software package (Partec $\mathrm{GmbH})$.

Electric cell-substrate impedance sensing (ECIS) adhesion and motility assay. The ECIS 1600R model instrument and 8W10 arrays (Applied Biophysics, Inc., Troy, NJ, USA) were used, as previously described (24). Following treatment of the surface with a cysteine solution, the arrays were incubated with complete medium for $1 \mathrm{~h}$. The same number of $\mathrm{HaCaT}$ cells $(40,000)$ was added to each well. After reaching confluence, the cells were subject to wounding using the integrated elevated field module in the instrument in the 1600R model (5 V, $30 \mathrm{sec})$. Changes in cellular impedance were recorded during initial attachment and following wounding at 4,000 Hz.

Statistical analysis. Experimental procedures were repeated independently a minimum of three times. Data were analysed using the Minitab 14 software, and statistical comparisons

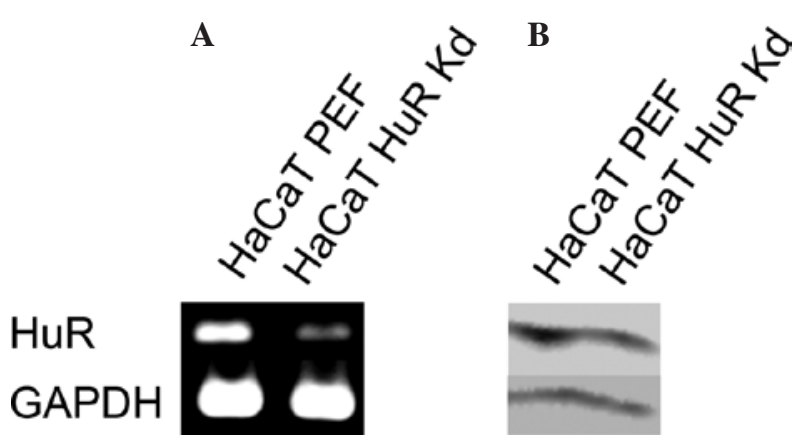

Figure 2. Creation of HuR knockdown keratinocytes. (A) Knockdown of HuR mRNA confirmed on standard PCR. (B) Knockdown of HuR confirmed with Western blotting. HuR Kd, HuR knockdown.

were drawn using the two-sample, two-tailed t-test or the Mann-Whitney non-parametric test as appropriate. $\mathrm{P}<0.05$ was considered to denote statistical significance.

\section{Results}

Expression levels of HuR transcripts in wound tissues. We firstly wanted to ascertain the expression of HuR in various tissue samples. Using a quantitative approach, we analysed the level of the HuR transcript in wound tissues using RT Q-PCR. Median levels of the HuR transcript were higher in healed chronic wounds [median (IQR): 0.044 (0-0.16)], compared to non-healed chronic wounds [median (IQR): 0 (0-1.0); Fig. 1], although this failed to reach statistical significance $(\mathrm{P}=0.13$, Mann Whitney U-test).

Knockdown of HuR expression in HaCaT cells. In order to further evaluate HuR function in vitro, we screened the human keratinocyte cell line for presence of HuR. After confirming that $\mathrm{HuR}$ was expressed in the HaCaT cells, we created a HaCaT HuR knockdown cell line and the control HaCaT PEF cell line (empty plasmid control) using stable transfection, as described above. After 2 weeks of cell culture in selection medium, mRNA was extracted from both cell lines. Standard PCR confirmed a reduction in HuR mRNA level in the HuR knockdown cell line compared to the PEF cell line, and subsequent Western blotting confirmed that HuR expression was also reduced at the protein level (Fig. 2).

Effect of HuR knockdown on growth, cell cycle and apoptosis. $\mathrm{HuR}$ has been shown to alter growth rates in cancer cell lines (25). After confirmation of knockdown, we analysed growth using a standard 5-day growth assay. When normalised to data from day $1, \mathrm{HuR}$ knockdown cells demonstrated a consistently faster rate of growth when compared to the PEF cell line $(\mathrm{P}<0.0001$; Fig. 3A).

In order to further clarify the mechanism via which HuR has its effect on growth, we analysed the effect of HuR knockdown on cell cycle and apoptosis. Cell cycle was analysed using the Partec CyFlow ${ }^{\circledR}$ SL flow cytometer. HuR knockdown resulted in a greater portion of cells progressing to the $\mathrm{S}$ and G2/M phase, compared to the PEF control (Fig. 3B). This would be consistent with the increased growth rate observed in the HuR knockdown cell line. 
A

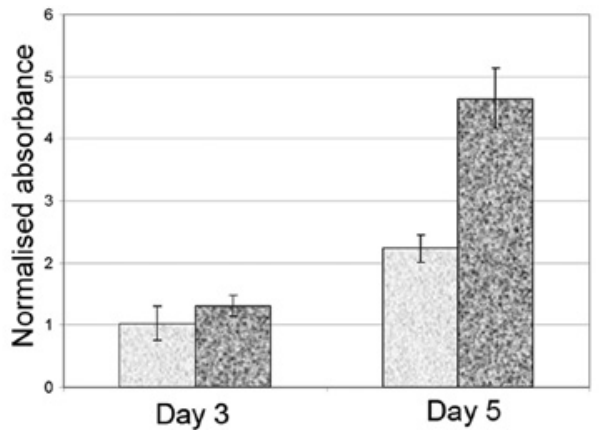

C

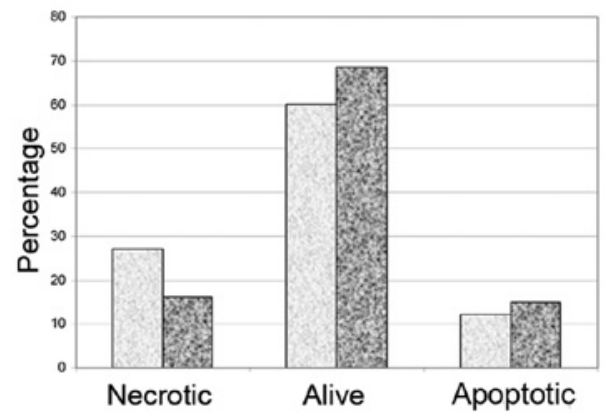

E

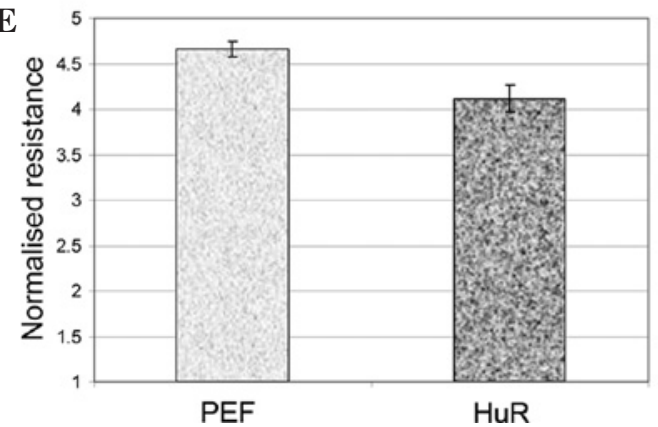

B

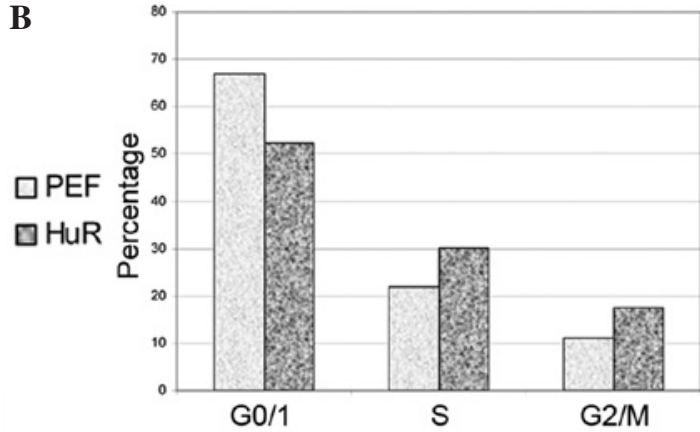

D

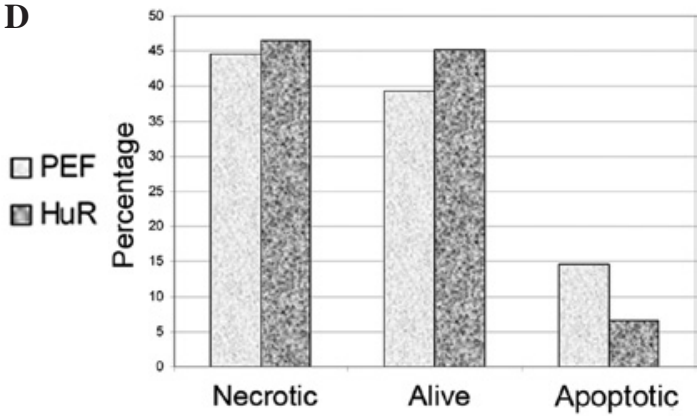

F

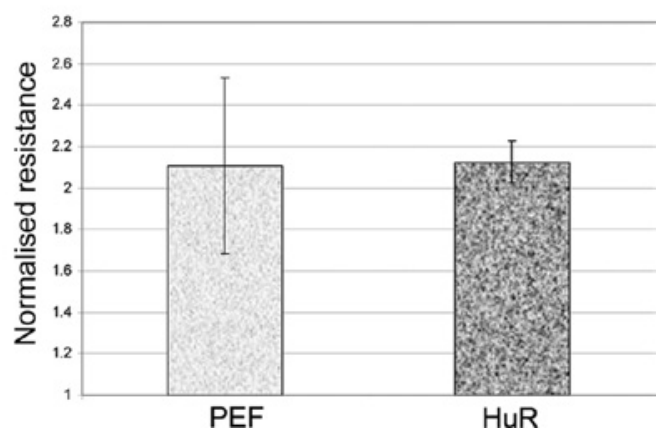

Figure 3. Effects of HuR knockdown on the biological functions of HaCaT and PEF cell lines (labelled HuR and PEF). (A) Growth assay normalised to day 1 to account for seeding bias. Results shown are the means of 3 repeats \pm SEM. There was no effect on growth rates on day 3 , but by day 5 there was significantly faster growth noted in the HuR knockdown cells compared to the PEF control ( $<<0.0001$, two-tailed t-test). (B) Analysis of cell cycle. Results from each cell line total 100\%. HuR knockdown resulted in a greater proportion of cells reaching G2/M phase, when compared to PEF. (C and D) Analysis of apoptosis with cells cultured in (C) normal medium and (D) serum-free medium. Results from each cell line total 100\%. HuR knockdown had minimal effect on apoptosis when cultured in normal medium, but showed a reduction in apoptosis in serum-free medium. This corresponds with an increase in the number of live cells As expected, necrosis is far more common in serum-free conditions compared to normal medium. (E) Analysis of adhesion at $4 \mathrm{~h}$ post-seeding. Results shown are the means of a minimum of 4 runs \pm SEM, and normalised to the intrinsic resistance within the ECIS machinery. HuR knockdown reduced adhesion significantly at $4 \mathrm{~h}(\mathrm{P}=0.02)$. (F) Analysis of post-wounding migration measured at $4 \mathrm{~h}$. Results shown are the means of a minimum of 4 runs \pm SEM, and normalised to the intrinsic resistance within the ECIS machinery. HuR knockdown had no significant effect on HaCaT migration.

We also analysed the effect of HuR knockdown on apoptosis. When cultured under normal conditions, HuR knockdown had minimal effect on apoptosis (Fig. 3C). When cultured in serum-free medium, HuR knockdown resulted in reduced levels of apoptosis, with a corresponding increase in live cells (Fig. 3D). Again, this would be consistent with the increased growth rate observed in the HuR knockdown cell line.

Analysis of adhesion and migration. We analysed the effect of HuR knockdown on both adhesion and migration using ECIS. Once cells were seeded, the internal resistance of each well was measured at regular intervals. As cells adhere to the electrode, resistance increases. We analysed normalised resistance at $4 \mathrm{~h}$, as shown in Fig. 3. This demonstrated a significantly reduced rate of adhesion of the HuR knockdown cell line ( $\mathrm{P}=0.02$; Fig. 3E).
After the resistance for each well had plateaued, typically after 6-8 h, the monolayer was wounded using the integrated elevated field module. We found that the optimum wound is created using $5 \mathrm{~V}$ for $30 \mathrm{sec}$ for each well. Following wounding, the resistance reaches its nadir, which is subsequently recorded as time zero, and against which all subsequent readings are normalised. We found that knockdown of HuR had no significant effect on rates of migration when compared to the PEF control ( $\mathrm{P}=0.16$; Fig. 3F).

\section{Discussion}

Successful wound healing requires the coordination of a number of processes to ensure timely closure of a wound. In recent years, greater attention has been given to molecular changes within keratinocytes on the edge of chronic wounds. 
Certain authors have explored variations in gene expression at the wound edge using microarray technology, searching for distinctive gene signatures and molecular patterns found in the keratinocytes bordering healing and non-healing wounds. For example, Cole et al (26) analysed the expression of mRNA in normal and acutely wounded skin from healthy volunteers at 30 and $60 \mathrm{~min}$ after wounding. They found that $124(3 \%)$ out of the 4,000 genes investigated (primarily involved in transcription and signalling) demonstrated a significant increase from baseline (2 times) at $30 \mathrm{~min}$ (with no genes significantly down-regulated). At $60 \mathrm{~min}, 46(1.15 \%)$ out of the 4,000 genes were significantly overexpressed, but 264 (6.6\%) out of the 4,000 genes (a number of which have unknown functions) were down-regulated. These findings help delineate the molecular changes occurring in the acute response to wounding. Other authors have used similar methods to evaluate the gene signatures of healing and non-healing wounds (27).

We recently investigated the effects of individual genes on wound healing using both in vitro and in vivo data. For example, Ehm2 (expressed in highly metastatic cells 2) was found to be up-regulated in acute wounds compared to chronic wounds. Further in vitro analysis showed that Ehm 2 promotes $\mathrm{HaCaT}$ motility, without affecting growth rates (28). Analysis of individual genes allows for a greater understanding of the molecular mechanisms behind the abnormal cellular behaviour noted in chronic wounds, and allows for identification of potential treatment targets.

HuR is a well-known post-transcriptional regulator of mRNAs rich in AREs (15). By shuttling these inherently unstable mRNAs from the nucleus to the cytoplasm, HuR affects a number of key cellular processes. Target mRNAs include the cell cycle regulators c-myc (29), cyclin A and B1 (30) and cyclin E1 (31), as well as angiogenic promoters and proto-oncogenes (15).

HuR has been extensively evaluated in cancer. Elevated levels have been associated with a poorer prognosis in a number of neoplastic processes, including colonic (10), breast (11), ovarian $(12,13)$ and gastric carcinoma (14). HuR expression appears to enhance the progression of cancer by up-regulating cellular proliferation, angiogenesis, invasion and migration (15). It is well-known that cancer and wound healing share a number of similar cellular changes, albeit occurring in an uncoordinated fashion in cancer $(32,33)$. We thus hypothesised that HuR would be up-regulated in healing wounds.

Our study is the first to investigate HuR with regards to wound healing using the HaCaT keratinocyte cell line. We showed that, as predicted, HuR expression was higher in chronic healed wounds compared to chronic non-healed wounds, although this failed to reach statistical significance. HuR knockdown increased growth in the HaCaT cell line, implying that elevated HuR levels reduce cellular growth. Furthermore, we demonstrated that this increased growth rate was due to more rapid progression through the cell cycle in the knockdown cell line, and a partial reduction in rates of apoptosis. When HuR expression is reduced in cancerous cell lines, growth is typically inhibited, as is cell cycle progression $(25,30)$. Thus, HuR knockdown results in markedly differing behaviour in the non-malignant $\mathrm{HaCaT}$ cell line compared to cancerous cell lines. Our research group has found similar differences between malignant and non-malignant cell lines with other proteins.
For example, Ehm 2 knockdown was found to inhibit growth in MCF-7 breast cancer cells by increasing apoptosis (34), whereas a similar knockdown in HaCaT cells had no effect on either growth or apoptosis (28). These data highlight the importance of caution when assuming that wound healing pathways function in an identical manner to cancerous pathways.

HuR knockdown reduces cellular adhesion, with minimal affect on migration. HuR thus functions as a pro-adhesive protein in the HaCaT cell line. Re-epithelialisation is characterized by epithelial migration, in which the adhesion of epithelial cells to the ECM plays a prominent role $(35,36)$. It is most likely that this effect on adhesion is the mechanism through which HuR promotes wound healing.

In conclusion, this is the first study to specifically analyse HuR in the context of wound healing. Elevated HuR levels are associated with a non-significant trend towards increased healing in vivo. We used a stably transfected HuR knockdown cell line to further evaluate the role of HuR in wound healing. In vitro data suggest that $\mathrm{HuR}$ reduces cellular growth, slows cell cycle progression and increases apoptosis. Furthermore, HuR improves cellular adhesion, a possible mechanism through which it enhances wound healing.

\section{Acknowledgements}

The authors wish to thank Mr. Kevin Conway for his help in the collection of wound tissue samples, and Ms. Fiona Ruge for her help with the processing of wound tissue samples. We thank the Welsh Government (A4B Programme) for supporting this study, and W.G.J. and Y.L. wish to thank the Cancer Research Wales for supporting this study.

\section{References}

1. Ma WJ, Cheng S, Campbell C, Wright A and Furneaux H: Cloning and characterization of $\mathrm{HuR}$, a ubiquitously expressed Elav-like protein. J Biol Chem 271: 8144-8151, 1996.

2. Ma WJ and Furneaux H: Localization of the human HuR gene to chromosome 19p13.2. Human Genet 99: 32-33, 1997.

3. Yao KM, Samson ML, Reeves R and White K: Gene elav of Drosophila melanogaster: a prototype for neuronal-specific RNA binding protein gene family that is conserved in flies and humans. J Neurobiol 24: 723-739, 1993.

4. Okano HJ and Darnell RB: A hierarchy of Hu RNA binding proteins in developing and adult neurons. J Neurosci 17: 3024-3037, 1997.

5. Ma WJ, Chung S and Furneaux H: The Elav-like proteins bind to AU-rich elements and to the poly(A) tail of mRNA. Nucleic Acids Res 25: 3564-3569, 1997.

6. Inoue M, Muto Y, Sakamoto H and Yokoyama S: NMR studies on functional structures of the AU-rich element-binding domains of $\mathrm{Hu}$ antigen C. Nucleic Acids Res 28: 1743-1750, 2000.

7. Beckel-Mitchener AC, Miera A, Keller R and PerroneBizzozero NI: Poly(A) tail length-dependent stabilization of GAP-43 mRNA by the RNA-binding protein HuD. J Biol Chem 277: 27996-28002, 2002.

8. Brennan CM and Steitz JA: HuR and mRNA stability. Cell Mol LifeSci 58: 266-277, 2001.

9. Bakheet T, Williams BR and Khabar KS: ARED 2.0: an update of AU-rich element mRNA database. Nucleic Acids Res 31: 421-423, 2003.

10. Heinonen M, Fagerholm R, Aaltonen K, et al: Prognostic role of HuR in hereditary breast cancer. Clin Cancer Res 13: 6959-6963, 2007.

11. Denkert C, Weichert W, Winzer KJ, et al: Expression of the ELAV-like protein HuR is associated with higher tumor grade and increased cyclooxygenase-2 expression in human breast carcinoma. Clin Cancer Res 10: 5580-5586, 2004. 
12. Denkert C, Weichert W, Pest S, et al: Overexpression of the embryonic-lethal abnormal vision-like protein HuR in ovarian carcinoma is a prognostic factor and is associated with increased cyclooxygenase 2 expression. Cancer Res 64: 189-195, 2004.

13. Erkinheimo TL, Lassus H, Sivula A, et al: Cytoplasmic HuR expression correlates with poor outcome and with cyclooxygenase 2 expression in serous ovarian carcinoma. Cancer Res 63: 7591-7594, 2003.

14. Mrena J, Wiksten JP, Thiel A, et al: Cyclooxygenase-2 is an independent prognostic factor in gastric cancer and its expression is regulated by the messenger RNA stability factor HuR. Clin Cancer Res 11: 7362-7368, 2005.

15. Yuan Z, Sanders AJ, Ye L and Jiang WG: HuR, a key posttranscriptional regulator, and its implication in progression of breast cancer. Histol Histopathol 25: 1331-1340, 2010.

16. Ather S, Chan D and Harding KG: The biology of wound healing. EJHP Practice 13: 53-54, 2007.

17. Harding KG, Morris HL and Patel GK: Science, medicine, and the future - healing chronic wounds. Br Med J 324: 160-163, 2002.

18. Nazarenko IA, Bhatnagar SK and Hohman RJ: A closed tube format for amplification and detection of DNA based on energy transfer. Nucleic Acids Res 25: 2516-2521, 1997.

19. Parr C and Jiang WG: Quantitative analysis of lymphangiogenic markers in human colorectal cancer. Int J Oncol 23: 533-539, 2003.

20. Jiang WG, Minhas U, Martin TA, Ruge F and Harding KG: Pattern of expression of CCN family members Cyr61, CTGF and NOV in human acute and chronic wounds. Exp Ther Med 2: 641-645, 2011.

21. Zuker M: Mfold web server for nucleic acid folding and hybridization prediction. Nucleic Acids Res 31: 3406-3415, 2003.

22. Jiang WG, Davies G, Martin TA, et al: Expression of membrane type-1 matrix metalloproteinase, MT1-MMP in human breast cancer and its impact on invasiveness of breast cancer cells. Int J Mol Med 17: 583-590, 2006.

23. Conway KP, Price P, Harding KG and Jiang WG: The role of vascular endothelial growth inhibitor in wound healing. Int Wound J 4: 55-64, 2007.

24. Chen J, Ye L, Zhang L and Jiang WG: Placenta growth factor, PLGF, influences the motility of lung cancer cells, the role of Rho associated kinase, Rock1. J Cell Biochem 105: 313-320, 2008.
25. Yuan Z, Sanders AJ, Ye L, Wang Y and Jiang WG: Knockdown of human antigen $R$ reduces the growth and invasion of breast cancer cells in vitro and affects expression of cyclin D1 and MMP-9. Oncol Rep 26: 237-245, 2011

26. Cole J, Tsou R, Wallace K, Gibran N and Isik F: Early gene expression profile of human skin to injury using high-density cDNA microarrays. Wound Repair and Regen 9: 360-370, 2001.

27. Kirsner RS, Charles CA, Tomic-Canic M, et al: A gene signature of nonhealing venous ulcers: potential diagnostic markers. J Am Acad Dermatol 59: 758-771, 2008.

28. Bosanquet DC, Ye L, Harding KG and Jiang WG: The role of EHM2 in wound healing. Br J Surg 98(S2): 23, 2011.

29. Lafon I, Carballes F, Brewer G, Poiret M and Morello D: Developmental expression of AUF1 and HuR, two c-myc mRNA binding proteins. Oncogene 16: 3413-3421, 1998.

30. Gorospe M, Wang WG, Caldwell MC, Lin SK and Furneaux H: HuR regulates cyclin A and cyclin B1 mRNA stability during cell proliferation. EMBO J 19: 2340-2350, 2000.

31. Hartley RS and Guo X: HuR contributes to cyclin E1 deregulation in MCF-7 breast cancer cells. Cancer Res 66: 7948-7956, 2006.

32. Werner S and Schafer M: Cancer as an overhealing wound: an old hypothesis revisited. Nat Rev Mol Cell Biol 9: 628-638, 2008.

33. Dvorak HF: Tumors: wounds that do not heal. Similarities between tumor stroma generation and wound healing. New Engl J Med 315: 1650-1659, 1986.

34. Jiang WG, Yu HF, Ye L, Mansel RE and Zhang YX: Clinical implications of the influence of Ehm2 on the aggressiveness of breast cancer cells through regulation of matrix metalloproteinase-9 expression. Mol Cancer Res 8: 1501-1512, 2010.

35. Suzuki K, Saito J, Yanai R, et al: Cell-matrix and cell-cell interactions during corneal epithelial wound healing. Prog Retin Eye Res 22: 113-133, 2003.

36. Kanchanawong P, Shtengel G, Pasapera AM, et al: Nanoscale architecture of integrin-based cell adhesions. Nature 468 580-584, 2010. 